\title{
Relación entre el desarrollo radicular de las terceras molares y la edad cronológica
}

\author{
Relationship between root development of third molars and \\ chronological age
}

\section{Resumen}

La estimación de la edad en individuos de 14 a 25 ańos es de utilidad para la identificación humana en la práctica clínica y tanatológica forense, así también, como indicador del crecimiento y desarrollo. Cuando todos los dientes permanentes han completado su formación, el desarrollo radicular de la tercera molar es el único indicador que puede ser utilizado para lograr predecir la edad de los individuos hasta los 25 años. El presente estudio piloto tiene como objetivo establecer la relación entre el desarrollo radicular de las terceras molares con la edad cronológica de los individuos comprendidos en este grupo etario. Para lograr este objetivo se contó con 25 terceros molares superiores e inferiores de pacientes de ambos sexos que ingresaron a la Clínica Odontológica de Universidad Nacional "San Luis Gonzága" de Ica. El desarrollo radicular de los dientes se estableció a través del patrón propuesto por Luis Toribio Suárez que divide el desarrollo radicular en diez etapas. La edad de los pacientes al momento de la extracción se expresó en edad decimal. Se efectuó la correlación entre ambas variables y se obtuvo una " $\mathrm{r}$ de Pearson" de 0,673. Asimismo, la diferencia media entre la edad dental con la edad cronológica fue de 1,42 ańos. Los resultados obtenidos meritan a realizar una investigación similar a la realizada en otros países que refleje la situación respecto a la población peruana con una muestra de mayor tamaño. Palabras clave: estimación de la edad dental, tercera molar, calcificación dental, odontología forense.

\begin{abstract}
The estimation of the age in individuals aged 14 to 25 years old is useful for human identification in the clinical practice and forensic thanatology, so also, as an indicator of growth and development. When all the permanent teeth have completed their formation, the third molar root development is the only indicator that can be used to predict the age of individuals up to 25 years. This pilot study aims to establish the relationship between root development of the third molars with the chronological age of the individuals included in this age group. To achieve this goal it was counted on 25 upper and lower third molars of patients of both sexes admitted to the Dental Clinic of National University of Ica "San Luis Gonzaga". Root development of teeth was established through the pattern proposed by Luis Suarez Toribio dividing root development in ten stages. The age of patients at the time of extraction was expressed as a decimal. The correlation between both of two variables was carried out obtaining a Pearson's " $r$ " coefficient of 0,673 . Also, the average difference between the dental age and the chronological age was 1.42. The results obtained warrant to conduct a research similar to that carried out in other countries reflecting the situation with respect to the Peruvian population with a larger sample size.
\end{abstract}

Key words: dental age estimation, dental calcification, forensic dentistry.

\section{Introducción}

La estimación de la edad biológica es un método muy utilizado en la atención médica y estomatológica especializada como un indicador clínico del crecimiento y desarrollo, asimismo, es utilizada en el campo de la medicina legal y la ciencia forense para fines de identificación humana. En este sentido, los peritos deben hallar los indicadores post-mortem que lleven a la identificación de un individuo: la edad, el sexo, la ascendencia, la estatura, los hábitos de lateralidad y las características individualizantes de todos aquellos cuya identidad se desconoce, sobre todo en los restos humanos esqueletizados. Una vez que se establecen estos indicadores se compara cada uno de ellos con los datos ante-mortem de la presunta persona(s) por identificar.

La estimación de la edad en cadáveres es un elemento definitorio para lograr la identidad del cuerpo, sobre todo cuando presenta un avanzado estado de descomposición, graves mutilaciones y/o quemaduras que van a determinar su condición de non nominatum (NN), es decir, un individuo sin identidad conocida. Para estos casos, la identificación debe valerse de los indicadores detallados en el párrafo precedente.
Artículo Original

\section{Carlos Alberto Suárez Canlla}

Odontólogo Forense

Correspondencia:

Dirección: Facultad de Odontología

Universidad Nacional "San Luis Gonzága” Ica Teléfono: 956791152

Correo electrónico: csc231274@yahoo.es
Fecha de recepción: 04-07-12

Fecha de aceptación: 26-11-12
En las personas, la estimación de la edad también es importante porque las ubica dentro de un grupo etario que le resulta de mucha utilidad para el magistrado al momento de tomar una decisión, sobre todo en aquellos(as) que están en tránsito a la edad adulta.

Para estimar la edad biológica se puede recurrir a diferentes procedimientos, el desarrollo dental es muy utilizado y su eficiencia es comparable con las que se apoyan en el crecimiento esqueletal. Tiene varias ventajas, entre ellas, la menor alteración cuando los tejidos son afectados por endocrinopatías u otras alteraciones del desarrollo humano (Smith 1991). ${ }^{1}$ 
Para estimar la edad biológica en restos humanos esqueletizados entre los 14 a 25 años de edad cronológica se utilizan varios indicadores: la erupción dental, la formación y calcificación de la corona y la raíz, la fusión de las epífisis con las diáfisis de los huesos largos, la fusión de segmentos de otros huesos, las características morfológicas de las carillas costales, las características morfológicas de la sínfisis púbica, entre otros. Sin embargo, acceder a cualquiera de estos indicadores (óseos) en cadáveres recientes NN resulta complicado ya que tendrían que disecarse los tejidos blandos de los alrededores, y por ende, la deformación del cadáver sería muy evidente y desagradable para los familiares. En el caso de las personas, el número de indicadores para establecer su edad resulta más limitado y se debe acceder a ellos a través de radiografías. Por tanto, de acuerdo a como se presenten los individuos y las condiciones de los mismos; el perito odontólogo deberá hacer uso de uno o más indicadores y los medios auxiliares para poder acceder a ellos. ${ }^{2,3,4}$

Para todos los casos, la formación y calcificación radicular de la tercera molar es el indicador más utilizado y de mayor accesibilidad. La tercera molar es la pieza dental cuya formación radicular se extiende desde los 14 a los 25 años de edad y, su relación entre su formación con la edad cronológica ha llevado a la formulación de muchos problemas de investigación en varios países. ${ }^{2,3,4}$

En España, la estimación de la edad de las personas reviste mayor importancia pues, los menores de 14 ańos se consideran inimputables (no responsables de sus actos). A las personas entre los $14 \mathrm{a}$ 18 años se les aplica la Ley del menor, que prevé un trato especial para los que cometen algún delito para las personas hasta los 21 años de edad pese a que son mayores de edad las leyes contemplan una reducción de la imputabilidad. ${ }^{5}$

En todos los grupos etarios y en cualquier región y/o país del mundo hallar la edad no solo implica aspectos relacionados a la identificación sino que, la experiencia y precisión de parte del perito será capaz de decidir el destino de esa persona en las esferas civil y penal. ${ }^{5}$

Mincer y cols evalúan la precisión de la estimación de la edad a partir del estado de desarrollo del tercer molar inferior, valorado según el método de Demirjian. Según este estudio, los estadios desde A a D - formación completa de la corona - y el estadio $\mathrm{H}$ - cierre completo apical - indicarían con una alta probabilidad que el individuo es mayor o menor de 18 ańos. El grado de exactitud, establecido en base a la diferencia entre la edad real y la calculada por el grado de desarrollo dentario, se sitúa en 4,8 años para el rango del $95 \%$ (dos desviaciones estándar). ${ }^{6}$

Thorson y Hagg utilizando el mismo sistema de valoración en una muestra de población sueca han observado una pobre relación entre la edad cronológica y la edad dental (infra estimación de la edad cronológica), con una diferencia media entre la edad estimada y cronológica de unos +/- 4.5 años en niñas (95\% de intervalo de confianza) y $+/-$ 2.8 años en niños, y un error intra-observador de $+/-0.8$ ańos (95\% intervalo de confianza) lo que inhabilita para los autores este método para la estimación de la edad.?

Kullman y cols obtienen valores similares aplicando un sistema basado en siete estadios de desarrollo de la raíz, con desviaciones estándar de 01 a 02 años sobre la edad media. ${ }^{8}$

En la revisión de Ritz-Timme et al, el tercer molar arroja un valor de error estándar que varía en un rango de 01 a 2.5 años, con coeficientes de correlación (r) entre 0.32 y 0.85 .

Toribio Suárez, L. odontólogo forense del Instituto de Medicina Legal de La Habana - encuentra una "r de Pearson" de 0.75 al correlacionar las variables desarrollo radicular de la tercera molar y edad cronológica. Cifra que en términos estadísticos otorga una buena correlación entre las variables antes citadas. La diferencia con los estudios citados anteriormente se refieren a la forma de medición de la variable formación y calcificación radicular de la tercera molar. Toribio S. utiliza dientes in vivo, no utiliza radiografías - panorámicas o periapicales - que utilizan otros investigadores; más aun, este autor hace una división del desarrollo radicular en diez (10) fases. ${ }^{9}$ Esta metodología utilizada otorga una mejor correlación entre las variables: edad cronológica y desarrollo radicular de la tercera molar. ${ }^{10}$

La función lineal hallada por Toribio S. como solución pronostica de estimaciones en base al patrón propuesto por el autor fue la siguiente ecuación de la recta de regresión, calculada por el método de los mínimos cuadrados:

$\mathrm{Y}=14,9+0,8 \mathrm{X}$

Dónde:

$\mathrm{Y}=$ edad decimal, $\mathrm{y}$

$\mathrm{X}=$ estadio de calcificación

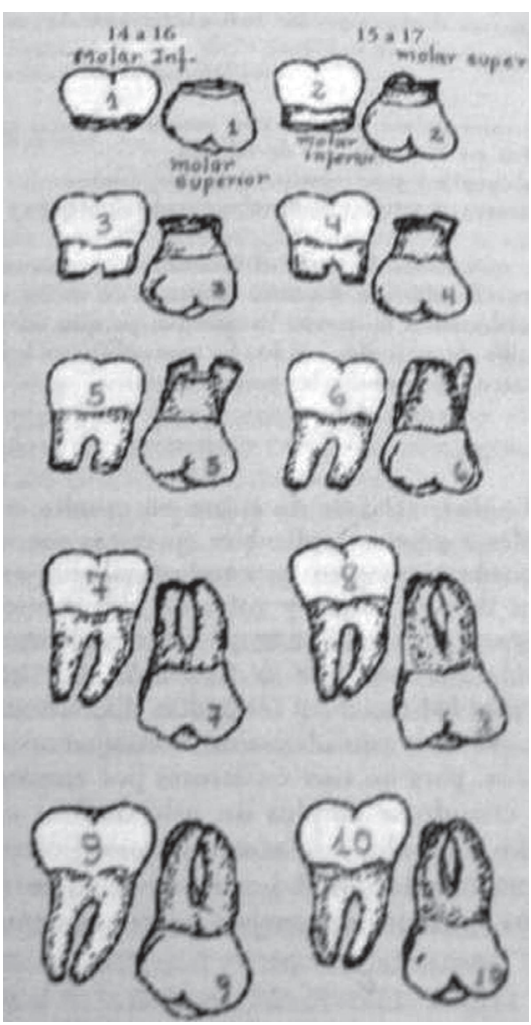

Fig 1. Patrón de clasificación de Luis Toribio Suárez.

El objetivo de este estudio es determinar la relación entre las etapas de formación y calcificación radicular de las terceras molares con la edad cronológica de personas de 14 a 25 años.

\section{Aspectos Embriológicos e Histológicos}

Dentro del período de campana avanzada se inicia la formación de la matriz del esmalte y la dentina, fenómenos conocidos como amelogénesis y dentinogénesis, seguidos por la cementogénesis, el desarrollo de la pulpa y el periodonto. Es así que, los responsables de la formación del esmalte son los ameloblastos y los responsables de formación de la dentina los odontoblastos; células encargadas de segregar la matriz dentinaria (predentina) necesaria para la formación de la dentina coronaria y

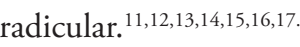

La dentinogénesis es el proceso de formación de la dentina, tanto coronal como radicular, que consta básicamente de dos momentos. El primer momento está relacionado a la secreción de la matriz orgánica, el segundo está relacionado a la calcificación de la misma. ${ }^{11,12,16}$

La formación de la dentina empieza en el estadio de campana avanzada en el lugar donde las células de la papila den- 
tal están adyacentes al epitelio dental interno. Desde este lugar, las células de la papila se diferencian en odontoblastos que se extienden por las paredes de las cúspides en dirección apical para ir engrosando lo que será posteriormente la dentina coronaria. ${ }^{11,12,15,16}$

Los odontoblastos se forman a partir del ectomesénquima de la papila dental. ${ }^{11,12,16}$ El proceso de diferenciación ocurre así: en las células del epitelio dental interno del órgano dental empiezan a producirse cambios en su forma, número y en sus organelas internas; estos cambios tienen relación con la preparación de dichas células en ameloblastos. Concomitante a esos cambios, o poco tiempo después, las células de la papila dental empiezan a diferenciarse en odontoblastos. Dichos cambios que se suscitan en el epitelio dental interno no ocurren en la vaina epitelial de Hertwig. Es preciso recordar la vaina de Hertwig está formada por el epitelio interno y externo del órgano dental, asimismo, solo después de que la vaina epitelial de Hertwig haya proliferado la dentina radicular se desarrollará. ${ }^{11,12,16}$

Cuando los odontoblastos han logrado su diferenciación empiezan a secretar una matriz orgánica - predentina - que se caracteriza por estar formada por fibras colágenas y sustancia fundamental amorfa; esta predentina está por debajo de la membrana basal en la que se apoya el epitelio dental interno. Todas estas primeras fibras colágenas y sustancia fundamental amorfa constituyen la matriz orgánica de la primera dentina o dentina del manto. Una vez secretada la predentina los odontoblastos empiezan a tener una actividad de fosfatasa alcalina a lo largo de sus membranas plasmáticas que se asocia al transporte de iones inorgánicos. ${ }^{11,12,15,16}$

Una vez que se deposita la primera capa de predentina los odontoblastos empiezan a secretar y dejar dentro de la predentina unas vesículas matriciales y unas prolongaciones odontoblásticas respectivamente. Las vesículas matriciales - que contienen los cristales de apatita -crecen, se rompen y se esparcen como un racimo de uva hasta unirse con otros cristalitos y lograr la mineralización de la matriz orgánica. Por otro lado, a los alrededores de las prolongaciones odontoblásticas también empiezan a colocarse las vesículas matriciales conteniendo los cristalitos de apatita para hacer posible la mineralización. ${ }^{11,12,16}$

Por otra parte, es necesario enfatizar que, la formación de dentina lleva un ritmo continuo y fásico, es decir, de producción y descanso, lo cual trae como consecuencia las líneas incrementales. En la corona puede depositarse hasta $08 \mu \mathrm{m}$. de dentina por día ${ }^{2}$ y en la raíz el depósito de dentina es más lento. ${ }^{11,12,15,16}$

La mineralización de la dentina se produce básicamente a través de tres patrones diferentes: lineal, globular y la combinación de los anteriores.

Respecto a este tema solo diremos que el proceso de calcificación general también es gradual, aunque en la región de la dentina peritubular resulta muy mineralizada desde un período muy temprano. ${ }^{11,12,15,16}$

Estos aspectos relacionados a la formación y calcificación de la dentina - sobre todo la dentina radicular - ocurren cuando los dientes están dentro de los maxilares, es decir, la mayor proporción de la formación de los dientes ocurre cuando ellos están dentro de sus criptas o alveolos. Esta característica del desarrollo dental lleva a una conclusión: existe una escasa o nula influencia del medio externo y, por el contrario, una mayor influencia genética durante el desarrollo de los dientes. ${ }^{11,12,15,16}$

Por otra parte, es importante destacar que los investigadores del tema en cuestión han hallado que el dimorfismo sexual es invertido: los hombres alcanzan los diferentes estadios de formación y calcificación dental - de la tercera molar - antes que las mujeres, independientemente de su origen étnico 5 .

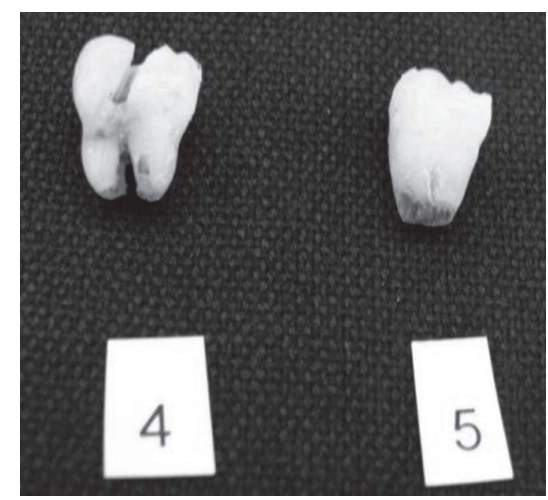

Fig 3. Distintos grados de formación radicular de terceros molares.

\section{Materiales y Método}

El estudio piloto contó con veinticinco terceras molares entre dientes superiores e inferiores - de pacientes de 14 a 25 años de edad de ambos sexos (15 varones y 10 mujeres). La muestra se obtuvo de las personas que ingresaron al servicio de Cirugía Bucal II de la Clínica Odontológica de la Universidad Nacional "San Luis Gonzága” de Ica.

Para conocer la verdadera edad de las personas se les solicitó su documento nacional de identidad (DNI). La edad de los pacientes se expresó en años, es decir; los días que forman las semanas, los meses y los ańos se expresaron en una sola unidad de medida anual. Para lograr este objetivo se recurrió a la edad decimal que consistió en dividir el año en diez partes en lugar de en doce. De esta forma, por ejemplo, la fecha decimal equivalente al 17 de agosto de 1998

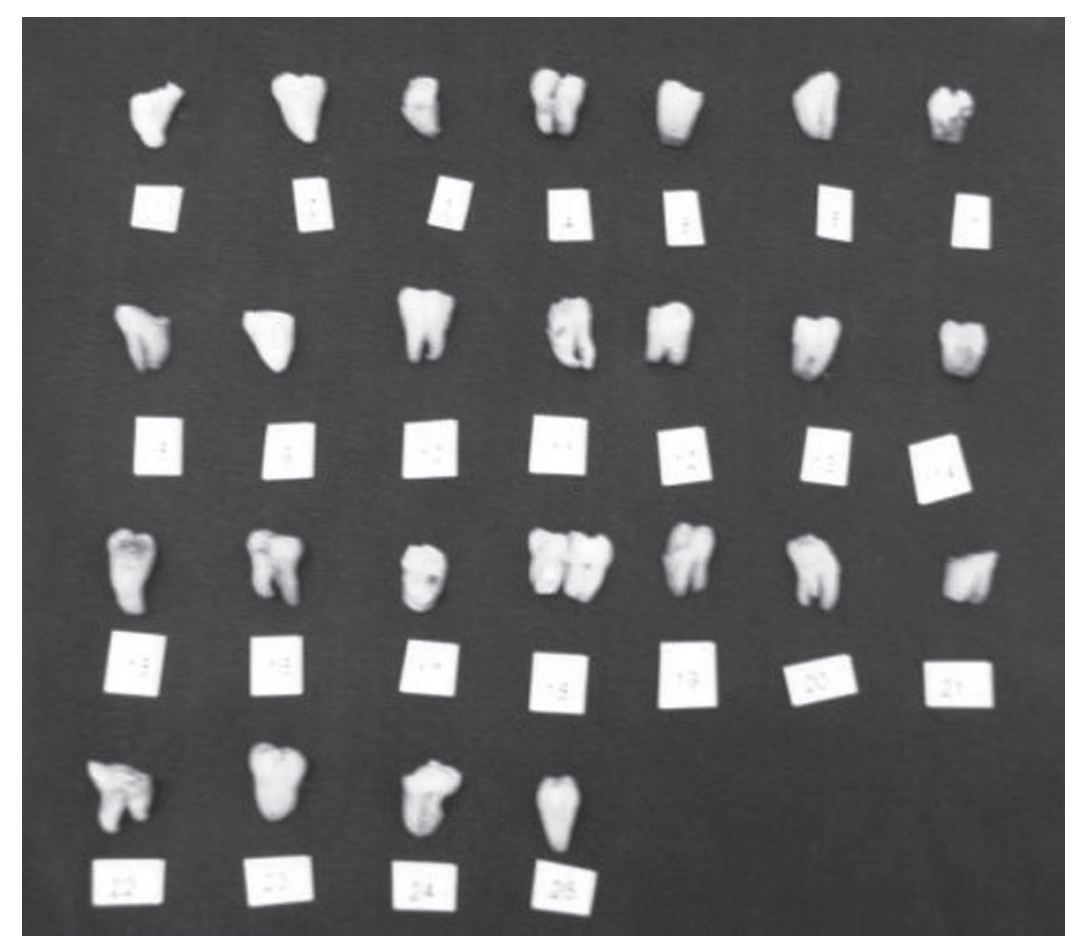

Fig 2. Muestra de estúdio. 
es el número 98.625 (donde 98 es el año y 625 el número correspondiente al día 17 de agosto). En tal sentido, la edad decimal del paciente al momento de la cirugía se calculó restando la fecha decimal del día de la extracción menos la fecha decimal del día de nacimiento. ${ }^{16}$ Para motivos prácticos se desarrolló una fórmula computarizada (en la hoja de cálculo excel) en la que se ingresó el equivalente decimal a la fecha de nacimiento y a la fecha de la cirugía. Los datos ingresados a la fórmula arrojan inmediatamente la edad decimal de la persona.

Las 25 piezas dentales se clasificaron de acuerdo al patrón propuesto por Toribio $S$. que va desde un trazo de formación radicular hasta la calcificación completa del periapice. En tal sentido, el patrón propuesto por el autor divide las etapas de formación radicular en diez estadios a los que les asigna los guarismos del 01 al 10 de acuerdo al desarrollo radicular (fig. 03). Debe tenerse presente que otros investigadores utilizan otras clasificaciones para relacionar el desarrollo dental con la edad cronológica, por ejemplo, la clasificación de Demirjian divide el desarrollo de un diente en ocho (08) estadios que van desde la letra "A hasta la H". $2,3,4,5,6,7,8,9,10$

Para establecer la asociación de las variables se realizó el análisis de correlación de Pearson. Asimismo, se procedió a medir la diferencia media entre la edad cronológica y la edad estimada a través del modelo propuesto.

La clasificación de las terceras molares se realizó a través de la observación y comparación directa (anatomía comparada) de cada uno de los dientes con el modelo propuesto. Resulta necesario mencionar que la comparación y clasificación se realizó "a ciegas", es decir, sin tener conocimiento de la edad de la persona al momento de la extracción, esto permitió evitar sesgos. La clasificación de cada una de las piezas dentales se registró en una base de datos elaborada para tal fin. Una vez que se obtuvo la base de datos se solicitó al profesional odontólogo (cirujano de la Clínica Odontológica de la Universidad) las fechas de intervención quirúrgica y las fechas de nacimiento de las personas que participaron en el estudio para hallar sus respectivas edades decimales.

La medición de la variable "formación y calcificación radicular" se tomó como una variable discontinua, es decir; a cada una de las piezas dentales de la muestra se le asignó un guarismo (del 01 al 10), de acuerdo al estadio de formación radicular que presentaba. La variable edad decimal se tomó como una variable continua, cuya expresión decimal fue producto de las conversiones de las fechas de nacimiento y de extracción a sus correspondientes fechas decimales.

\section{Resultados}

La edad promedio de las personas que participaron en la muestra fue 21.4 ańos y el rango de edad estuvo entre los 16.64 a 24.59 ańos de edad.

Se realizó el análisis de correlación que obtuvo una "r de Pearson" de 0,673. No se efectuó el análisis de regresión lineal simple debido a que la función lineal que pronostica la misma no ofrecería una fórmula confiable basada en una muestra de veinticinco (25) personas.

Por otra parte, la diferencia media entre la edad dental con la edad cronológica fue de 1.42 ańos. Solo cuatro piezas dentales tuvieron una diferencia elevada de 4.82; 2.12; 3.41 y 3.15 ańos.

En la tabla 1 se puede apreciar el desarrollo radicular de las veinticinco (25) terceras molares basado en el patrón de clasificación de Luis Toribio Suárez, asimismo, se puede observar la edad decimal de cada uno de las personas que participaron en el estudio piloto.

\section{Discusión}

El procesamiento estadístico de la variable formación y calcificación radicular fue tratado como una variable discontinua, durante la clasificación de las molares. A cada uno de los dientes de la muestra se le asignó un guarismo (del 01 al 10) de acuerdo al grado de desarrollo que presentaba. Investigaciones relacionadas al desarrollo radicular de las terceras molares y la edad cronológica utilizan las clasificaciones de Demirjian, Moorrees y, Gleiser y Hunt para medir el desarrollo de las mismas. Todas ellas emplearon el mismo tratamiento estadístico que les permitió hallar la " $\mathrm{r}$ de Pearson" y las ecuaciones que predicen la edad de las personas a partir de su desarrollo radicular.

El índice de correlación de Pearson obtenido en el presente estudio $(0,673)$ es similar a otros hallados en investigaciones relacionadas al tema, esto demuestra que existe una relación entre la edad cronológica de las personas y el desarrollo radicular de sus terceras molares que merece resaltarse.

La variable edad cronológica expresada como edad decimal de cada una de las personas de la muestra de estudio permitió trabajar de manera más precisa, sencilla y con una sola unidad de medida. En este sentido, la conversión de las fechas de nacimiento y de extracción a sus correspondientes fechas decimales constituyó un paso previo de importancia en el tratamiento de esta variable.

La desviación estándar hallada (1.42 años) también es similar a otras investigaciones como las reportadas por Mincer $\mathrm{HH}$, Mesotten K. que no permiten establecer de manera certera si una persona es mayor o menor de edad. En tal sentido, una investigación que cuente con una muestra de mayor tamaño ofrecería datos más exactos y mejores datos probabilísticos al momento de decidir - por parte del magistrado - si

Tabla No 01: Desarrollo radicular de las terceras molares de las personas de la muestra de acuerdo al patrón propuesto por Luis R. Toribio Suarez y la edad decimal de cada uno de ellos.

\begin{tabular}{ccccccccccccc}
$\mathbf{N}^{0}$ & $\mathbf{1}$ & $\mathbf{2}$ & $\mathbf{3}$ & $\mathbf{4}$ & $\mathbf{5}$ & $\mathbf{6}$ & $\mathbf{7}$ & $\mathbf{8}$ & $\mathbf{9}$ & $\mathbf{1 0}$ & $\mathbf{1 1}$ & $\mathbf{1 2}$ \\
\hline D.R. & 6 & 5 & 7 & 5 & 6 & 7 & 4 & 7 & 7 & 7 & 8 & 5 \\
\hline E.D. & 21.81 & 17.54 & 22.48 & 19.48 & 21.48 & 19.81 & 19.16 & 21.8 & 21.95 & 20.9 & 20.92 & 19.71 \\
& & & & & & & & & & & & \\
13 & 14 & 15 & 16 & 17 & 18 & 19 & 20 & 21 & 22 & 23 & 24 & 25 \\
\hline 6 & 8 & 8 & 7 & 6 & 6 & 8 & 9 & 5 & 7 & 8 & 9 & 8 \\
\hline 16.64 & 23.32 & 23.5 & 24.59 & 18.19 & 23.33 & 23.36 & 22.6 & 22.3 & 23.28 & 23.28 & 21.55 & 22.18
\end{tabular}

No: número de personas.

DR: desarrollo radicular.

ED: edad decimal. 
una persona tiene la condición de ser mayor de edad.

\section{Conclusiones}

- Los resultados obtenidos revelan la existencia de una correlación entre el desarrollo radicular de la tercera molar y la edad cronológica de los pacientes que participaron en el estudio piloto.

- Los resultados obtenidos muestran la misma tendencia de investigaciones relacionadas al desarrollo radicular de las terceras molares y la edad cronológica que cuentan con muestras de mayor tamaño.

\section{Recomendaciones}

1. Los resultados obtenidos motivan a recopilar un mayor número de piezas dentales o radiografías que constituyan una muestra representativa que arroje resultados más confiables que puedan ser utilizados por los peritos dedicados a temas de identificación. En este sentido, una investigación basada en una muestra peruana tendría mejor certeza al momento de estimar la edad - sobre todo en las personas en tránsito a la edad adulta - que a la vez serviría como punto de comparación con otras investigaciones que se han desarrollado en otros países.

2. Las muestras basadas en películas radiográficas - físicas o digitales - deberían estar alrededor de las mil (1000) radiografías panorámicas u ortopantomografías ya que investigaciones similares recopilan un número de radiografías alrededor de esta cifra. Si la muestra estuviera basada en dientes in vivo, esta no debería ser menor a doscientos sesenta y cuatro (264) piezas dentales, cifra utilizada por Toribio $S$. para su estudio.

\section{Referencias bibliográficas}

1. Rodríguez JV. La antropología forense en la identificación humana. Bogotá: Guadalupe Ltda; 2004. 113-114 p.

2. Gleiser I. Hunt E. The permanent mandibular first molar: its calcification, eruption and decay. American Journal of Physical Anthropology 2005 Abr; 13(2): 282-3 p.

3. Willems G. A review of the most commonly used dental age estimation techniques. The Journal of Forensic Odonto-Stomatology 2001 Jun; 19(1): 10-1 p.

4. Mesotten K. Gunst K. Carbonez A. Willems G. The Journal of Forensic Odonto-Stomatology 2003 Dec; 21(2): 33 p.

5. Prieto JL. La maduración del tercer molar y el diagnóstico de la edad. Evolución y estado actual de la cuestión. Cuad. Med. Forense 2008 Ene; 14(51): 12-13 p.

6. Mincer $\mathrm{HH}$, Harris EF, Berryman HE (1993) The A.B.F.O. study of third molar development and its use as an estimator of chronological age. J For Sci 38(2):379-90.

7. Thorson J, Hagg U (1991) The accuracy and precision of the third mandibular molar as an indicator of chronological age. Swed Dent J 15(1):15-22.

8. Kullman L, Johanson G, Åkesson L (1992) Root development of the lower third molar and its relation to chronological age. Swed Dent J 16:161-7.

9. Ritz-Timme S, Cattaneo C, Collins MJ, Waite ER, Schutz HW, Kaatsch HJ, Borrman HI (2000) Age estimation: the state of the art in relation to the specific demands of forensic practise. Int J Legal Med 113(3):129-36.

10. Toribio S. Estimación de la edad por los terceros molares en subadultos y adultos jóvenes (tesis doctoral). La Habana: Instituto Superior de Ciencias Médicas de la Habana Facultad "Calixto García"; 1999. 66-8 p.

11. Bhaskar SN. Histología y embriología bucal de Orban. 90 ed. Washington: El Ateneo; 1986. 144-150 p.

12. Ten Cate AR. Histología oral. $2^{a}$ ed. Princeton: Panamericana; 1986. 100, 182-188 p.

13. Di Fiore M. Diagnostico histológico. Buenos Aires: El Ateneo; 1965. $365 \mathrm{p}$.

14. Eliséiev BG. Afanasieb YI. Yúrina NA. Histología. Danilova IV, traductor. Moscú: Mir Moscú; 1985. 439-441 p.

15. Hillson S. Dental anthropology. New York: Cambridge University Press; 1996. 372 p.

16. Bascones A. Tratado de odontología - tomo IV. 2a ed. Madrid: Avances medico dentales; 1998.4680 p.

17. Perea PB, Sánchez SJ, Domínguez GS. Antropología y paleontología dentarias. Madrid: MAPFRE; 2002. 482 p. 